\title{
The Radio-FIR Correlation in the Milky Way
}

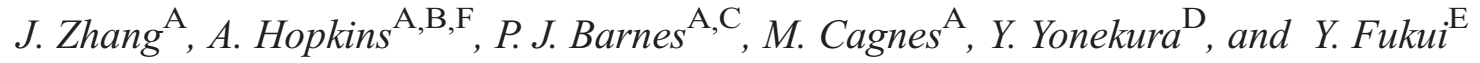 \\ A Sydney Institute for Astronomy, School of Physics, University of Sydney, NSW 2006, Australia \\ B Anglo-Australian Observatory, PO Box 296, Epping, NSW 1710, Australia \\ ${ }^{C}$ Astronomy Department, University of Florida, Gainesville, FL 32611, USA \\ D Faculty of Science, Ibaraki University, 2-1-1 Bunkyo, Mito, Ibaraki 310-8512, Japan \\ ${ }^{\mathrm{E}}$ Department of Astrophysics, Nagoya University, Furo-cho, Chikusa-ku, \\ Nagoya 464-8602, Japan \\ F Corresponding author. Email: ahopkins@aao.gov.au
}

Received 2008 December 22, accepted 2010 June 13

\begin{abstract}
We investigate the scale on which the correlation arises between the $843 \mathrm{MHz}$ radio and the $60 \mu \mathrm{m}$ far-infrared (FIR) emission from star forming regions in the Milky way. The correlation, which exists on the smallest scales investigated (down to $\approx 4 \mathrm{pc}$ ), becomes noticeably tight on fields of size $30^{\prime}$, corresponding to physical scales of $\approx 20-50 \mathrm{pc}$. The FIR to radio flux ratio on this scale is consistent with the radio emission being dominated by thermal emission. We also investigate the location dependence of $q_{\text {mean }}$, a parameter measuring the mean FIR to radio flux ratio, of a sample of star forming regions. We show that $q_{\text {mean }}$ displays a modest dependence on galactic latitude. If this is interpreted as a dependence on the intensity of star formation activity, the result is consistent with studies of the Large Magellanic Cloud (LMC) and other nearby galaxies that show elevated values for $q$ in regions of enhanced star formation.
\end{abstract}

Keywords: Galaxy: general — galaxies: evolution — galaxies: spiral — galaxies: starburst — infrared: galaxies — radio continuum: galaxies

\section{Introduction}

The tight correlation between radio and far-infrared (FIR) emission in external galaxies has been long known (e.g., Yun et al. 2001). To explain the correlation, the conventional picture suggests that both emissions are the result of massive star formation. The radio emission has both a thermal component and a non-thermal synchrotron component due to cosmic ray electrons accelerated to relativistic speeds through supernovae shocks. The FIR is mostly thermal emission from dust excited by UV emission associated with star formation. The FIR emission is a common measure of star formation rates (SFRs, Kennicutt et al. 1998), although a contribution to the FIR emission from older stellar populations (Bell et al. 2003) necessitates a more complex calibration from luminosity to SFR than a simple linear relation. The effects of different dust geometries and opacities in the immediate vicinity of different star forming regions potentially adds a significant complication (Calzetti et al. 1995; Calzetti 1997). There is also a strong trend for higher levels of dust obscuration to occur preferentially in galaxies of higher SFR (e.g., Hopkins et al. 2001; Afonso et al. 2003; PérezGonzález et al. 2003), so it is not unreasonable to expect a similar variation in individual star forming regions.

A complication in understanding the radio-FIR correlation arises from the fact that synchrotron radiation occurs on a larger spatial scale, due to its diffusion length, than the FIR thermal emission (see, e.g., Murphy et al. 2006). Measurements of the total FIR to radio flux ratio, $q$ (Helou et al. 1985), for the Milky Way (Broadbent et al. 1989) show that on the largest scales our Galaxy is consistent with other star forming galaxies, although these authors also emphasise that the tight global correlation is not seen within galaxies. Measurements of $q$ within a few hundred pc around Orion (Boulanger \& Perault 1988) give a value elevated compared to the average for star forming galaxies, and similar to those seen in regions of active star formation in the Large Magellanic Cloud (LMC) by Hughes et al. (2006) and within nearby galaxies (Murphy et al. 2006). A question of interest then becomes, on what spatial scale does the radio-FIR correlation begin to appear around star forming regions? It has been proposed that this should start to appear on scales comparable to the $\approx 1-2 \mathrm{kpc}$ diffusion length for cosmic ray electrons (Bicay \& Helou 1990; Marsh \& Helou 1998). This would correspond to the observed region around an area of massive star formation being large enough to include sufficient quantities of radio synchrotron emission. We show that, for the Milky Way, a radio-FIR correlation exists on much smaller scales, and is likely to be a correlation between the thermal radio and FIR components.

Recent studies of the radio-FIR correlation have concentrated on analysing the physical processes which lead to the correlation on galactic scales. This has been done by analysing the correlation on small spatial scales $(\mathrm{Xu}$ et al. 1992; Hughes et al. 2006), and within other nearby galaxies (Murphy et al. 2006). Hughes et al. (2006), looking at the Large Magellanic Cloud (LMC), achieved 
a spatial resolution of $20 \mathrm{pc}$. On such fine spatial scales, these authors have demonstrated varying $q$ for different structures in the LMC. In regions of the most active star formation, $q$ is higher. This is interpreted as due to two different physical mechanisms driving the radio-FIR correlation. In the most active star forming regions, radio emission is mostly thermal while in less active regions, the correlation is due to synchrotron radiation. We compute the mean $q$, called $q_{\text {mean }}$ herein, for star forming regions grouped by their galactic latitude. We show that $q_{\text {mean }}$ is elevated closer to the galactic plane, where the most active star formation in our galaxy is occuring, compared to the value at higher galactic latitudes.

\section{Analysis}

We investigate the spatial scale on which the radio-FIR correlation begins to arise around massive star forming regions in the Milky Way. We then extend this analysis to explore whether the radio-FIR correlation shows any Galactic latitude dependence, as might be expected if $q$ depends on star formation rate, as suggested by the elevated $q$ values seen in the LMC bar (Hughes et al. 2006) and spiral arms of nearby galaxies (Murphy et al. 2006).

\subsection{Data}

The star forming regions used for this analysis are drawn from the Census of High and Medium-mass Protostars (CHaMP) survey conducted with the Mopra dish of the Australia Telescope ${ }^{1}$ (Barnes et al. 2006, 2010a). CHaMP targeted massive dense gas clumps based on the Nanten Survey (Yonekura et al. 2005) of carbon monoxide gas, a standard tracer of the sites of star formation. The CHaMP catalogue is based on an examination of complete Nanten maps for the region $280^{\circ}<l<300^{\circ},-4^{\circ}<b<+2^{\circ}$, made in four $J=1-0$ molecular spectral lines in the $3 \mathrm{~mm}$ band: $\mathrm{CO},{ }^{13} \mathrm{CO}, \mathrm{C}^{18} \mathrm{O}$, and $\mathrm{HCO}^{+}$(see also Yonekura et al. 2005 , for a subset of these data). The CHaMP catalogue contains 209 local emission maxima in $(l, b, v)$ space, identified from the Nanten $\mathrm{C}^{18} \mathrm{O}$ and $\mathrm{HCO}^{+}$data cubes (Barnes et al. 2010b). In this sense, the CHaMP catalogue comprises an unbiased sample of all the dense gas clumps in this window, and is therefore a useful indicator of the most likely sites of current massive star formation in this part of the Milky Way. The unbiased nature of the CHaMP selection, allowing identification of all massive star forming regions throughout a representative spiral arm of the Milky Way, suggests that our analysis should be generally applicable to the Galaxy as a whole.

Using the coordinates of the 173 highest CO luminosity sources from the CHaMP survey, being those already catalogued at the initiation of this study, 138 target regions were selected for this analysis based on

\footnotetext{
${ }^{1}$ The Mopra telescope is part of the Australia Telescope which is funded by the Commonwealth of Australia for operation as a National Facility managed by CSIRO. The University of New South Wales Digital Filter Bank used for the observations with the Mopra Telescope was provided with support from the Australian Research Council.
}

availability of both radio and FIR images. The omission of the $20 \%$ of targets lacking radio and FIR data should not introduce any significant bias to our analysis, as we still sample the full range of Galactic latitude and longitude within the CHaMP survey area. The radio images at $843 \mathrm{MHz}$ were obtained from the Sydney University Molonglo Sky Survey (SUMSS, Bock et al. 1999; Mauch et al. 2003) and FIR images at $60 \mu \mathrm{m}$ from the Infrared Astronomical Satellite (IRAS) Sky Survey Atlas (ISSA) ${ }^{2}$. An advantage of the SUMSS radio images for this analysis is the high sensitivity to extended emission, a consequence of the closely-spaced and highly redundant spacings between receivers in the Molonglo Observatory Synthesis Telescope (Bock et al. 1999). The resolution of the SUMSS images is $45^{\prime \prime} \times 45^{\prime \prime} \operatorname{cosec}|\delta|$, and the internal flux density scale is accurate to within 3\% (Mauch et al. 2003). An example with both SUMSS and IRAS images is given in Figure 1, showing a region two degrees on a side, centred on one of the CHaMP targets.

\subsection{The Spatial Scale on Which the Correlation Arises}

For each CHaMP star forming region, SUMSS and ISSA images of varying sizes were obtained. Square field sizes of $5^{\prime}, 10^{\prime}, 15^{\prime}, 30^{\prime}$, and $1^{\circ}$ were analysed. Regions $2^{\circ}$ on a side were also obtained, but most of the SUMSS images of this size were incomplete. This is a limitation of the SUMSS image server, which does not mosaic adjacent images if the requested field extends beyond the boundary of the nearest $4^{\circ} \times 4^{\circ}$ archival image. To measure the correlation between the FIR and radio images of the same star forming region, a simple flux sum was taken by adding up the amplitudes of all pixels within the image. Figure 2 shows radio flux density as a function of FIR flux for the four smallest spatial scales measured. Each of the 138 star forming regions is shown, except for those fields where the flux summed to a negative value in the radio images due to the presence of artifacts. Such fields were not included in any of our analyses.

The correlation coefficient was calculated for each field size (Table 1). A significant correlation is found for all, even the smallest field sizes. By fields of size $30^{\prime} \times 30^{\prime}$ this correlation becomes visibly tight, especially for the brightest sources (associated with high SFRs). The correlation at the smallest scales $\left(5^{\prime}\right.$ to $\left.30^{\prime}\right)$ was tested using FIR images randomly offset from the radio images. The correlation coefficients found for these non-associated regions are significantly reduced, as would be expected if the correlation is due to the emission from the massive star forming regions. For the randomly offset fields, the correlation coefficient varies from 0.61 for the $5^{\prime}$ fields to 0.64 at $15^{\prime}$, increasing slightly to 0.75 at $30^{\prime}$. This supports the suggestion that, even on the smallest scales measured here, there is a measurable radio-FIR correlation associated

\footnotetext{
${ }^{2}$ This research has made use of the NASA/IPAC Infrared Science Archive, which is operated by the Jet Propulsion Laboratory, California Institute of Technology, under contract with the National Aeronautics and Space Administration.
} 

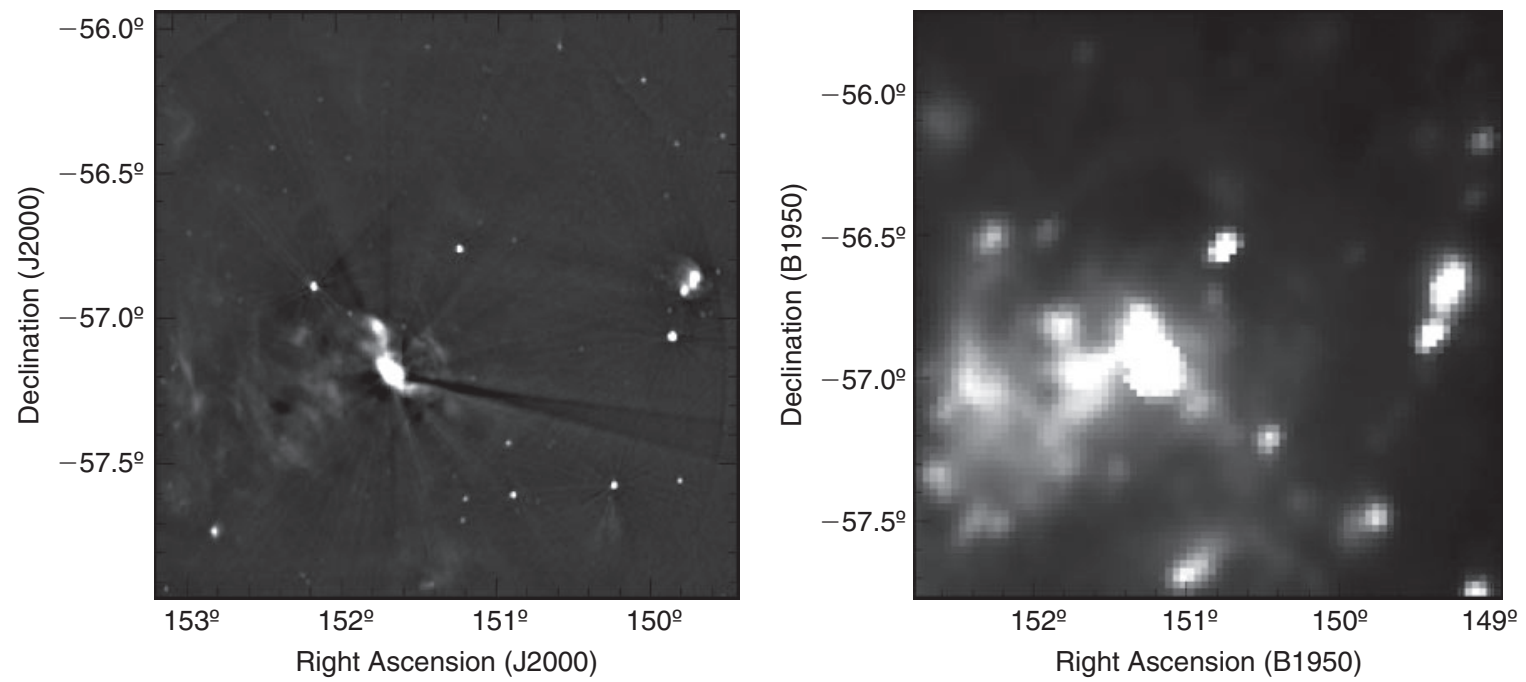

Figure 1 SUMSS $843 \mathrm{MHz}$ (left) and IRAS $60 \mu \mathrm{m}$ (right) images for a $2^{\circ} \times 2^{\circ}$ region centred on one of the target CHaMP massive star forming regions.
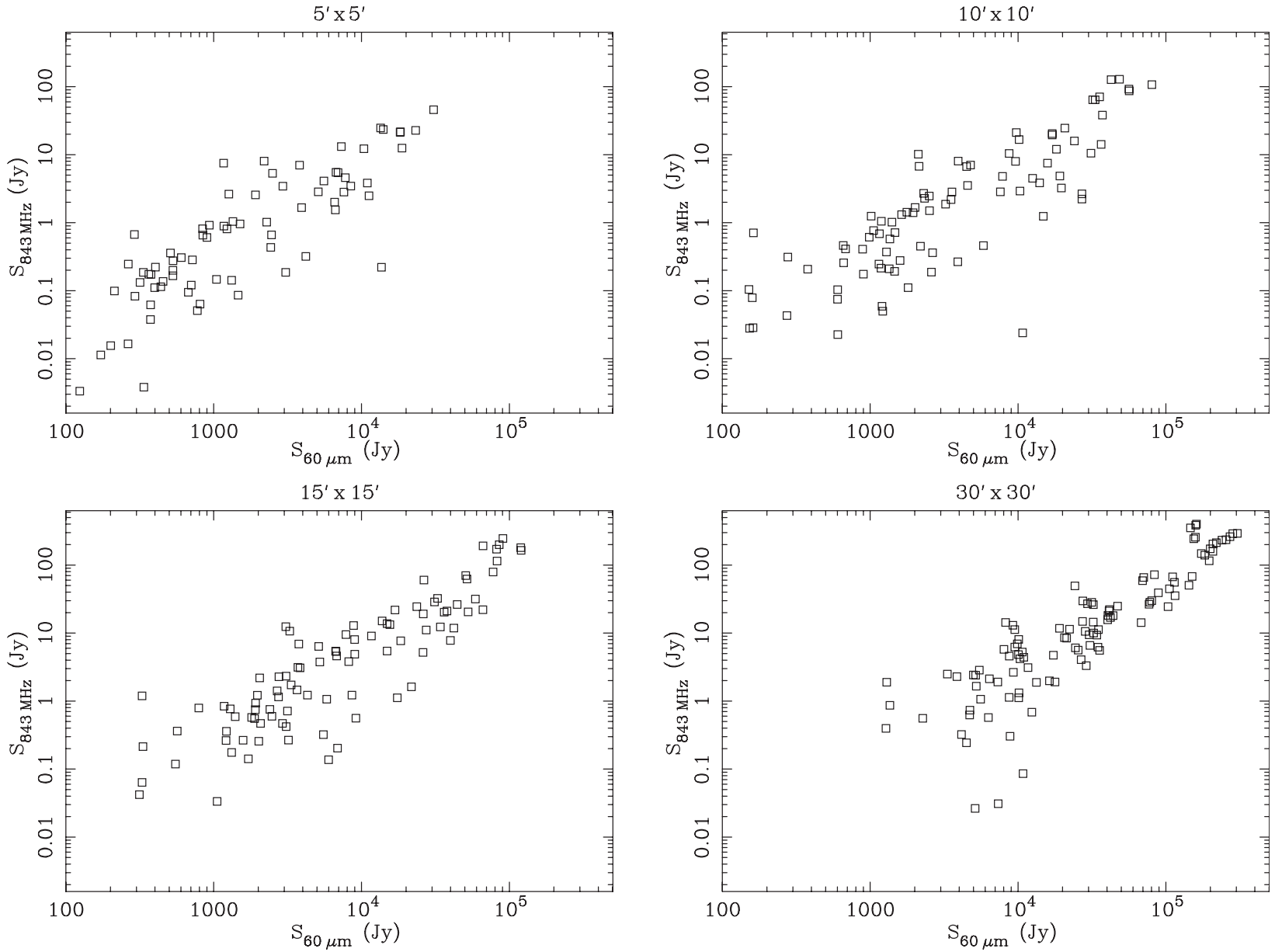

Figure 2 SUMSS $843 \mathrm{MHz}$ radio flux density as a function of IRAS $60 \mu \mathrm{m}$ flux, for regions of progressively larger size centred on CHaMP massive star forming regions. The radio-FIR correlation tightens noticeably once the area analysed reaches about $30^{\prime} \times 30^{\prime}$. This corresponds to a physical scale of $\approx 20 \mathrm{pc}$ to $50 \mathrm{pc}$.

with the CHaMP massive star forming regions. A value for $q_{\text {mean }}$ was calculated, along with the corresponding rms scatter in the individual $q$ values for each field. The observed rms scatter in $q$ for our targets is consistent with the ranges of $q$ found within galaxies by Hughes et al. (2006) and Murphy et al. (2006). It is likely that much of this observed scatter is real and not simply a result of limitations in our approach, or due to measurement 
uncertainty. The carbon monoxide isotopologues, used as a tracer by the CHaMP survey, identify the locations of the coldest and densest gas clumps. Such dense gas clumps are variously associated with pre-stellar gas, active star forming regions, and residual clouds from recent massive star formation. Such variation could account for a significant portion of the scatter observed in $q$.

In order to ensure these measurements provide robust estimates of $q_{\text {mean }}$, we explored several possible sources of bias: (1) The presence of artifacts in the radio images; (2) The contribution of a non-zero background level in the ISSA images; (3) Overlapping fields, arising from the clustering of CHaMP sources. For each of these tests the values of $q$ and $q_{\text {mean }}$ were remeasured using the $30^{\prime} \times 30^{\prime}$ images, and the correlation coefficient recalculated to ensure no bias was introduced. The initial correlation coefficient found for the $30^{\prime} \times 30^{\prime}$ images was 0.87 . Images removed in the artifact analysis include those displaying radial artifacts originating from bright sources, and those with large negative-flux regions associated with bright sources, along with images that did not encompass the full field of view as a consequence of the SUMSS image archive limitations mentioned above. Excluding those radio images visually identified as containing artifacts marginally decreases the correlation coefficient to 0.84 . It was noted that the rms scatter of $q$ in the sample with visually identified artifacts removed was not significantly changed, indicating that such artifacts are unlikely to be the primary source of the observed scatter. The median background level in each IRAS image was measured from the histogram of pixel intensities, and subtracted prior to calculating $q_{\text {mean }}$. This resulted in only minor changes to the resulting $q$ values as the background level was low compared to the total flux. The resulting correlation coefficient is 0.85 . To identify the impact of image overlap, $q_{\text {mean }}$ was calculated only for images independent by more than $75 \%$ of their size. The resulting correlation coefficient is 0.74 , possibly suggesting that overlapping fields account for some of the strong correlation seen in Table 1. A smaller fraction of the $15^{\prime}$ fields, though, are similarly overlapping, and removing those does not significantly change the correlation coefficient found (0.86) compared to the full sample $(0.87)$ for that field size. We conclude that our estimate of the field size on which the radio-FIR correlation arises is not sensitive to these limitations.

Table 1. The correlation between the FIR and radio emission around regions identified in the CHaMP survey in the Milky Way. Values of $q_{\text {mean }}$ and the rms scatter in individual $q$ values are also shown

\begin{tabular}{lccc}
\hline $\begin{array}{l}\text { Field size } \\
(\operatorname{arcmin})\end{array}$ & $\begin{array}{l}\text { Correlation } \\
\text { coefficient }\end{array}$ & $q_{\text {mean }}$ & $\begin{array}{c}\text { rms } \\
\text { scatter }\end{array}$ \\
\hline 5 & 0.86 & 3.41 & 0.54 \\
10 & 0.83 & 3.34 & 0.54 \\
15 & 0.87 & 3.33 & 0.47 \\
30 & 0.87 & 3.42 & 0.48 \\
60 & 0.81 & 3.56 & 0.44 \\
\hline
\end{tabular}

To infer the spatial scale associated with this angular size we require the distances to the CHaMP star forming regions. Although there are currently only a limited number of distances available, we make use of those in hand to estimate the length scale on which the radio-FIR correlation arises. For our sample of 138 star forming regions, there are 54 distance measurements, based on a combination of techniques. Some of the objects have distance estimates based on nearby or embedded HII regions, while most are estimated kinematically. The $(l, v)$ diagrams in the direction of our sample fields are largely unambiguous, with distance uncertainties of $\approx 10-20 \%$. Less than $50 \%$ of the distance measurements suffer from a near/far ambiguity. Using these estimates, we can identify the approximate range on which the radio-FIR correlation begins. An angular scale of $30^{\prime}$ corresponds to a spatial scale of $22 \mathrm{pc}$ to $59 \mathrm{pc}$ at the estimated distances of $2.5 \mathrm{kpc}$ to $6.8 \mathrm{kpc}$. This reflects an approximate diameter centred on the star forming regions. The $5^{\prime}$ field sizes, on which a correlation also exists, correspond to (diameter) scales of $3.6 \mathrm{pc}$ to $9.9 \mathrm{pc}$. These scales are all significantly smaller than the diffusion length scale expected for cosmic ray electrons (Bicay \& Helou 1990), and support the idea that correlations between different components of the radio and FIR emission exist over a variety of spatial scales. This result will be explored further in the discussion. In the next section we quantify our measurement of $q_{\text {mean }}$ and explore its galactic latitude dependence.

\subsection{Galactic Latitude Dependence}

The average $60 \mu \mathrm{m}$ FIR to $843 \mathrm{MHz}$ radio ratio of selected images is measured by the $q_{\text {mean }}$ parameter and is defined as:

$$
q_{\text {mean }}=\left\langle\log _{10}\left(S_{60 \mu \mathrm{m}} / S_{843 \mathrm{MHz}}\right)\right\rangle .
$$

Note that this differs from the $q$ typically defined in that here we use different FIR wavelength and radio frequency. This definition of $q_{\text {mean }}$ will not affect the trends observed. The use of $843 \mathrm{MHz}$ rather than $1.4 \mathrm{GHz}$ radio measurements will act to reduce our $q_{\text {mean }}$ by up to 0.15 (for pure synchrotron emission) compared to that of Hughes et al. (2006). This estimate simply assumes a conversion from $843 \mathrm{MHz}$ to $1.4 \mathrm{GHz}$ using a spectral index $\alpha=-0.7$ (with $S \propto v^{\alpha}$ ). Using the $60 \mu \mathrm{m}$ flux only, rather than the combined $60 \mu \mathrm{m}$ and $100 \mu \mathrm{m}$ FIR flux reduces our estimate of $q_{\text {mean }}$ by approximately 0.15 again, estimated assuming that on average $S_{100 \mu \mathrm{m}} \approx$ $1.6 S_{60 \mu \mathrm{m}}$, and using the definition of FIR of Helou et al. (1985). This total systematic offset of $\approx 0.3$ is slightly smaller than the rms scatter in our measurement (Table 1).

Values for $q_{\text {mean }}$ are shown in Table 1. After correcting the FIR images by subtracting the background level, we find $q_{\text {mean }}=3.34$ for the $30^{\prime}$ fields, and $q_{\text {mean }}=3.50$ for the $1^{\circ}$ fields, only marginally smaller than the initial estimates, given the observed scatter. The values that we find here for $q_{\text {mean }}$, especially after considering the systematic offset of $\approx 0.3$ indicated above, correspond to 
flux ratios more than an order of magnitude larger than those corresponding to the typical value of $q \approx 2.3$ found for star forming galaxies (e.g., Condon 1992). Our estimates are, however, consistent with the elevated values of $q$ identified within nearby galaxies in regions of elevated star formation (Hughes et al. 2006; Murphy et al. 2006).

To measure any possible galactic latitude dependence of $q_{\text {mean }}$, we use the $1^{\circ}$ images, although the results are similar with other field sizes. The images were divided into two bins of galactic latitude, $|b| \leq 0.8^{\circ}$ and $|b|>0.8^{\circ}$, containing 39 and 32 target regions respectively. The value of $q_{\text {mean }}$ shows a clear galactic latitude dependence, having higher values on average closer to the galactic plane (Figure 3). A Kolmogorov-Smirnov (KS) test comparing the $q_{\text {mean }}$ values for the two subsamples gives $P<1 \times 10^{-3}$, indicating that they are inconsistent with having been drawn from the same population. The $S_{60 \mu \mathrm{m}}$ flux values for each of these subsamples were also compared using a KS test. This shows again that, with $P<1 \times 10^{-3}$, the $60 \mu \mathrm{m}$ fluxes of the subsamples are inconsistent with being drawn from the same population, and that those at lower latitudes show higher $60 \mu \mathrm{m}$ fluxes consistent with having higher star formation rates.

\section{Discussion}

The first main result of this investigation is that a local radio-FIR correlation seems to arise at spatial scales of at least $\approx 20-60 \mathrm{pc}$, and likely on scales as small as a few pc. The second main result is that the FIR-radio ratio $(q$ mean $)$ has a galactic latitude dependence. We interpret this second result to indicate $q$ is dependent on the level of star formation activity.
The scale on which the radio-FIR correlation appears around star formation regions is consistent with the LMC study (Hughes et al. 2006) where the correlation arises at $\approx 20 \mathrm{pc}$ and becomes tight above $\approx 50 \mathrm{pc}$. We further find a correlation on even smaller scales, down to $\approx 4 \mathrm{pc}$. The diffusion length of relativistic electrons contributing to the galactic FIR-radio ratio $(\approx 1-2 \mathrm{kpc}$, Bicay \& Helou $1990)$ is much larger than these spatial scales. This suggests that the correlation on such a local scale is between thermal radio and FIR, an interpretation consistent with other studies showing strong correlation between thermal radio and warm dust emission (Hoernes et al. 1998; Hughes et al. 2006). The thermal fraction at $843 \mathrm{MHz}$ calculated from Equation 5 of Condon (1992) is about 0.08 , so assuming that the FIR emission is constrained to the locale of the star forming region, the flux ratios observed would be expected to be about an order of magnitude larger than those corresponding to $q \approx 2.3$ found as the average for star forming galaxies in general. This is consistent with the values that we have measured, supporting the result that the high values of $q_{\text {mean }}$ from this study, as well as earlier estimates (Boulanger \& Perault 1988), are a consequence of not including the synchrotron radio emission that is distributed on larger scales.

The result of the latitude dependence analysis is consistent with a dependence of $q_{\text {mean }}$ on the star formation rate. This is based on the assumption that star formation rates are higher closer to the galactic plane, supported by the increased $S_{60 \mu \mathrm{m}}$ measured there. Our results are consistent with those of Hughes et al. (2006), who observed elevated $q$ values in the bar of the LMC where there is elevated star formation activity. Strong additional

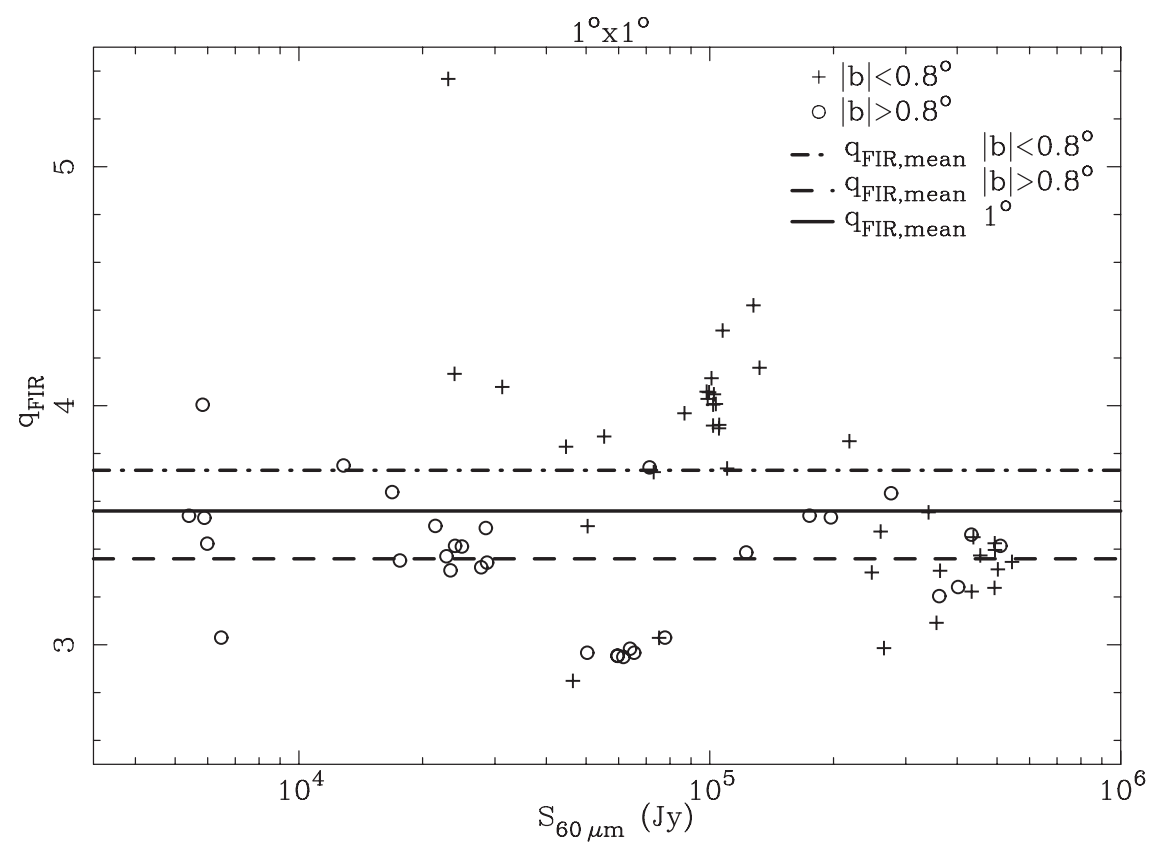

Figure 3 Values of $q$ as a function of $S_{60 \mu \mathrm{m}}$ for the two subsamples split by distance from the Galactic plane. $q_{\text {mean }}$ is elevated closer to the Galactic plane, where the star formation, indicated by $S_{60 \mu \mathrm{m}}$, is most active. 
support for our intepretation of the galactic latitude analysis comes from a study of local spiral galaxies. Murphy et al. (2006) observed that active star forming regions of galaxies with high SFR display elevated $q$ values.

From Table 1, the change in $q_{\text {mean }}$ between the different spatial scales is unlikely to be statistically significant given the rms scatter in the measurements. Even out to scales of $2^{\circ}$ (spatial scales of up to $\approx 200 \mathrm{pc}$ ), where these were able to be measured, the value of $q_{\text {mean }}$ remains essentially unchanged, although there is perhaps a very marginal elevation on scales of $30^{\prime}$ to $1^{\circ}$. This, while not statistically significant, may imply a suggestive trend. We suggest that a rise in $q_{\text {mean }}$ at $\approx 20-50$ pc and the decrease on larger scales around star forming regions could be due to the various components of FIR and radio emission occuring on different spatial scales. The change in $q_{\text {mean }}$ as spatial scale increases is then a consequence of the changing proportions of these different components. We can describe such a behaviour with a simple model for the distribution of these components consistent with the known spatial scales of each emission process.

On the smallest scale, thermal FIR and radio are dominant. The thermal radio emission is due to free-free emission in ionised gas while the thermal FIR emission is from hot dust. On a slightly larger scale, cirrus FIR emission continues to contribute, eventually ceasing and leaving only the synchrotron radiation component on the largest scale.

The simple model shown in Figure 4 demonstrates that any rise in $q_{\text {mean }}$ between the $30^{\prime}$ and the $1^{\circ}$ images could be due to the cirrus FIR emission continuing to greater spatial scales than the thermal radio emission. The value of $q$, of course, is calculated using fluxes corresponding to the integral of these intensities within a given scale. On the kiloparsec scale, and for a single star forming region, $q$ would continue to decrease with the increasing contribution of the synchrotron radiation, since all the FIR emission has ceased. Within galaxies, the overlapping of adjacent star forming regions on scales comparable to the synchrotron diffusion length then gives rise to the observed lower average value of $q$ dominated by the synchrotron component.

This model, focussing on the dependence of $q$ on spatial scale, does not directly address whether or not $q$ should be higher around a region of higher star formation rate, for the same spatial scale sampled (Figure 3). This may be the case, for example, if the relative proportion of thermal FIR and radio emission is itself dependent on the star formation rate.

\section{Conclusion}

We have examined the scale on which the radio-FIR correlation arises around star forming regions in the Milky Way. The correlation is tight by scales of $\approx 20-60 \mathrm{pc}$, and exists on scales even as small as $\approx 4 \mathrm{pc}$. The galactic latitude dependence of $q_{\text {mean }}$ is consistent with that found in the LMC and local spiral galaxies (Hughes et al. 2006; Murphy et al. 2006), and strongly suggests that $q$ is elevated in more active star forming regions. We interpret this as a correlation between the thermal radio emission and the FIR.

We have suggested a simple model that accounts for these observed trends in $q_{\text {mean }}$. To test this model, we plan to compare individual $q$ values, on a range of spatial scales, with the directly measured star formation rates in each CHaMP target region. Star formation rates can be

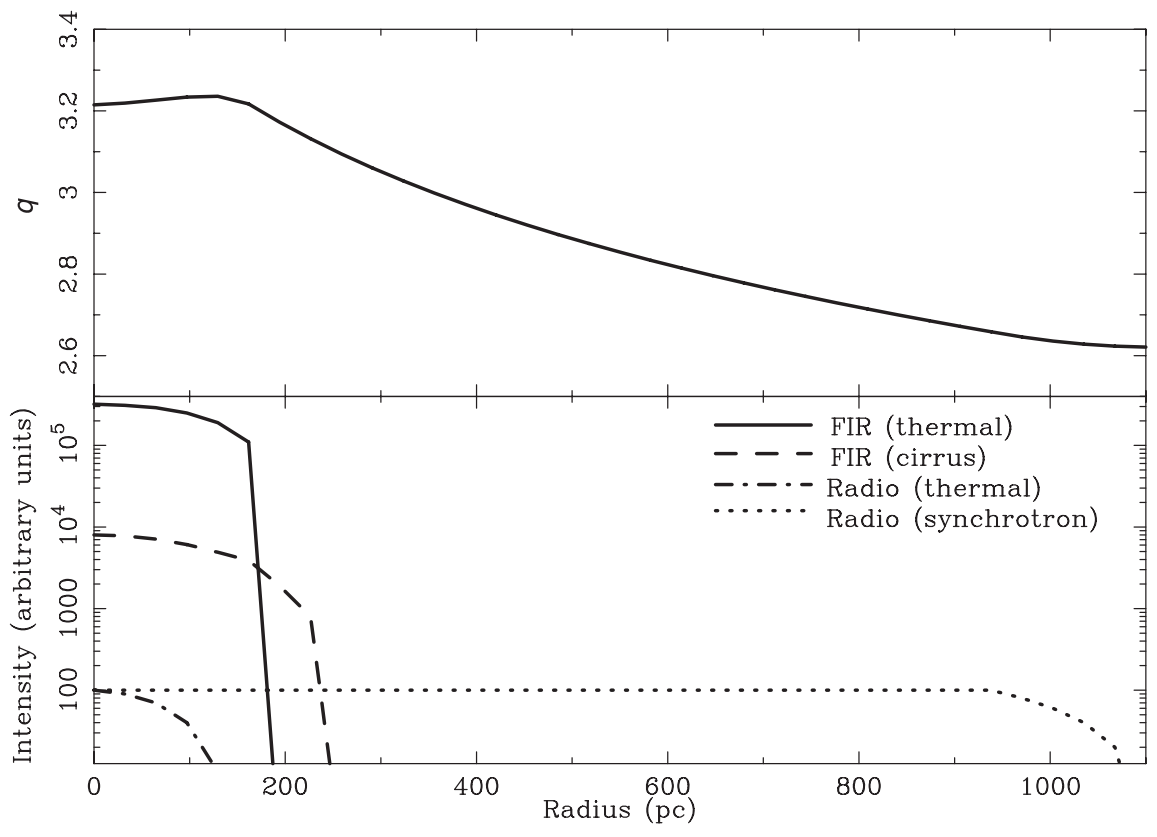

Figure 4 A simple model of the radial scale on which the components of FIR and radio emission extend, centred on a star forming region. 
measured in a number of different ways, including the radio and FIR luminosities measured specifically to encompass each star formation region. For such radio measurements, which are likely to be primarily thermal emission, existing star formation rate calibrations for total radio luminosity can be revised by referring to the thermal to total radio flux ratios of Condon (1992). H $\alpha$ luminosities, as well, may be able to be obtained for many of the regions, and used in estimating star formation rates. Additional data for the CHaMP targets is also planned to be obtained through programs on Gemini-S, SOFIA, and Herschel.

Multifrequency radio continuum measurements for each star forming region would allow determination of the thermal-to-synchrotron ratio as a function of spatial scale. This would provide a more accurate quantitative constraint on the scales within the simple model proposed here. It would also allow a refinement of the radio-derived star formation rates.

\section{Acknowledgments}

We warmly thank the referee for extensive and thorough comments that have led to significant improvements in this paper. A.M.H. acknowledges support provided by the Australian Research Council through a QEII Fellowship (DP0557850). P.J.B. acknowledges support through NSF grant AST-0645412 at the University of Florida.

\section{References}

Afonso, J., Hopkins, A., Mobasher, B. \& Almeida, C., 2003, ApJ, 597,269
Barnes, P. J. et al., 2006, in IAU Symposium 231: “Astrochemistry Throughout the Universe”, Eds. Lis, D., Blake, G. A. \& Herbst, E. (Cambridge UP: Cambridge)

Barnes, P. J., Yonekura, Y., Ryder, S. D., Hopkins, A. M., Miyamoto, Y., Furukawa, N. \& Fukui, Y., 2010a, MNRAS, 402, 73

Barnes, P. J. et al., 2010b (in preparation)

Bell, E., 2003, ApJ, 586, 794

Bicay, M. D. \& Helou, G., 1990, ApJ, 362, 59

Bock, D., Large, M. I. \& Sadler, E. M., 1999, AJ, 117, 1578

Boulanger, F. \& Perault, M., 1988, ApJ, 330, 964

Broadbent, A., Osborne, J. L. \& Haslam, C. G. T., 1989, MNRAS, 237,381

Calzetti, D., 1997, in "The Ultraviolet Universe at Low and High Redshift: Probing the Progress of Galaxy Evolution", Eds. Waller, W. H. et al. (New York) AIP Conf. Proc. 408, 403

Calzetti, D., Bohlin, R. C., Kinney, A. L., Storchi-Bergmann, T. \& Heckman, T. M., 1995, ApJ, 443, 136

Condon, J. J., 1992, ARA\&A, 30, 575

Helou, G., Soifer, B. T. \& Rowan-Robinson, M., 1985, ApJ, 298, L7

Hoernes, P., Berkhuijsen, E. M. \& Xu, C., 1998, A\&A, 334, 57

Hopkins, A. M., Connolly, A. J., Haarsma, D. B. \& Cram, L. E., 2001, AJ, 122, 288

Hughes, A., Wong, T., Ekers, R., Staveley-Smith, L., Filipovic, M. Maddison, S., Fukui, Y. \& Mizuno, N., 2006, MNRAS, 370, 363

Kennicutt, R. C. Jr, 1998, ARAA, 36, 189

Marsh, K. A. \& Helou, G., 1998, ApJ, 445, 599

Mauch, T., Murphy, T., Buttery, H. J., Curran, J., Hunstead, R. W., Piestrzynska, B., Robertson, J. G. \& Sadler, E. M., 2003, MNRAS, 342, 1117

Murphy, E. J. et al., 2006, ApJ, 651, L111

Pérez-González, P. G., Zamoranoy, J., Gallego, J., AragónSalamanca, A. \& Gil de Paz, A., 2003, ApJ, 591, 827

$\mathrm{Xu}$, C., Klein, U., Meinert, D., Wielebinski, R. \& Haynes, R. F., 1992, A\&A, 257, 47

Yonekura, Y. et al., 2005, ApJ, 634, 476

Yun, M. S., Reddy, N. A. \& Condon, J. J., 2001, ApJ, 554, 803 